\title{
Model Test on Failure Modes of Anchorage Tunnels Subjected to Blast Loading
}

\author{
Xu Jingmao ${ }^{1,2, *}$, Gu Jincai ${ }^{2}$, Chen Anmin ${ }^{2}$, Zhang Xiangyang ${ }^{2}$ and Wang Tao ${ }^{2}$ \\ ${ }^{1}$ Department of Modern Mechanics, University of Science and Technology of China, Hefei, Anhui 230027, China \\ ${ }^{2}$ Luoyang Institute of Hydraulic Engineering Technology, Luoyang, Henan 471023, China
}

Received 21 June 2018; Accepted 8 September 2018

\begin{abstract}
The premise of anti-explosion reinforcement design of underground tunnels is to find out the failure form and failure mechanism of anchorage tunnels to take targeted reinforcement measure sunder explosion load. However, the antiexplosion reinforcement effect of bolts(cables) on underground tunnels is seldom studied at present, and the failure law of tunnels is not clear, which cannot provide scientific basis for anti-explosion reinforcement design. In view of this, the model test apparatus for anti-explosion structures in geotechnical engineering and explosive load simulation test technology were firstly developed in this study, and based on Froude similarity theory, a series of anti-explosion model tests on different anchorage tunnels subjected to blast loads, including plane charge and mass charge, were carried out. The failure modes of several kinds of anchorage tunnels under explosion loads were obtained. Some conclusions were drawn from the model test results. Under the same test conditions, spalling occurs in tunnel roof in the plane charge test, while shear cracks and stagger cracks occur in tunnel spandrel in the mass charge test. The anti-explosion capacity of the tunnel reinforced by full-grouted bolts is better than that by part-grouted bolts. The augmentation of bolt length has no control effect on preventing crack extension in surrounding rock, when bolt spacing decreases to a certain density, it just prevents cracks from extending into the reinforced region. The local and dense lengthening bolts in tunnel spandrel can prevent or interdict cracks from extending in surrounding rock, or compel cracks to detour the reinforced region; consequently, it can enhance anti-explosion capacity of the tunnel. This study provides a significant reference for improving reinforcement measures of the underground anti-explosion structures and its anti-explosion capacity.
\end{abstract}

Keywords: Geotechnical engineering, Explosion load, Reinforced tunnel, Model test

\section{Introduction}

Bolt (cable) reinforcement, as an effective and active rock reinforcement measure [1-2], has been widely used in civil engineering and national defense projects, such as the coal mine tunnel, underground powerhouse and storage cavern. Based on the previous researches, many scholars have basically clarified the reinforcement mechanism, design and calculation method of bolts(cables) under static loading, but with the development of modern precision guided drill ground weapons [3-5], the underground anchorage tunnel will be confronted with the danger of explosion load. The stress waves generated by explosion, mainly including spherical wave and plane wave, propagate in the rock mass and interact with the surrounding rock and supporting structure of the tunnel, the propagation rule and the mechanism of action are extremely complex, which is obviously different from the static mechanical behavior under the action of geotectonic stress, and the destruction of the tunnel also shows obvious dynamic characteristics, such as collapse, spallation and punching.

In order to carry out the reasonable and effective antiexplosion reinforcement design of underground tunnel, it is necessary to find out the failure form and failure mechanism of anchorage tunnel under explosion load, so as to take targeted reinforcement measures. However, the present

\footnotetext{
*E-mail address: ximtwty@sina.com

ISSN: 1791-2377 С 2018 Eastern Macedonia and Thrace Institute of Technology. All rights reserved. doi:10.25103/jestr.114.15
}

analytical solutions of wave problem in geotechnical anchorage are confined to the propagation rules of elastic wave in anchor bolt, geotechnical medium and their coupling system [6], which are only applicable to the finite range in some idealized environment, and the failure modes of anchorage tunnels cannot be obtained. The experimental studies that have been conducted also focus on the dynamic response of bolts, such as dynamic strain, prestress variation, and so on [7-8]. And the macroscopic failure phenomena of the tunnel obtained from the experiment are also under certain conditions.

Therefore, it is necessary to systematically study the failure modes of anchorage tunnels under explosive loading, and obtain the damage rules and damage sites of the tunnels under different explosion loads and different reinforcement schemes, so as to put forward corresponding reinforcement measures to strengthen the key sites. It is of great significance to design the anti-explosion reinforcement scheme and improve the anti-explosion ability.

\section{State of the art}

To study the safety of underground tunnels under explosive load has important social, economic and military significance. Anchor bolt is a very effective technical means to reinforce underground tunnel. A large number of scientific research and engineering practice show that the underground tunnel reinforced by anchor bolts has good static load resistance. However, the study of its dynamic 
load resistance is still in the preliminary stage. So far, scholars all over the world have carried out a great deal of research on the anti-explosion performance of underground anchorage tunnels by means of theoretical analysis, experimental and numerical simulation methods, and some dynamic response laws of anchorage tunnels have been given. However, these researches have mainly focused on the dynamic mechanical properties of bolts, the propagation law of explosion wave in bolts and rock mass, and dynamic stress process in surrounding rock. Such as Yang and Wang [6] performed a theoretical research on the leakage characteristics of wave energy in the bolt, and the elastic wave propagation in the bolt, rock and their coupling system. The attenuation property and propagation mechanism of the wave in the anchorage system were gained. Through field tests, Tannant et al. [7] studied the dynamic characteristics of end anchoring bolt under blast loading, as well as the dynamic strain of bolts and the velocity of rock surface, and pointed out that the influence factors of blast loading on the pre-stressed bolt are explosion pulse amplitude, duration and load cycle. Yang et al. [8] studied the change of anchor prestress in the course of explosion by model test. Ortlepp and Stacey [9] conducted a research on reinforcement measures of large-deformation tunnel under static and dynamic loading, and pointed out that the yielding bolt can absorb large amounts of energy without causing damage to the tunnel. Yi and $\mathrm{Lu}$ [10] analyzed the influence of blasting vibration on cement mortar bolt through theoretical research. Zhang, Xue, and Rong et al. [11-13], used numerical simulation method to study the mechanical response of the anchor under blasting stress wave respectively. Zeng, Xiao, and $\mathrm{Cao}$ et al. [14-16] studied the anti-explosion performance of spray-anchor support and soil nailing support in the loess tunnel. The mechanical characteristics of spray-anchor support, the pressure distribution of surrounding rock and the critical resistance of soil nailing support were investigated. Ren [17] analyzed and compared the measured stress responses, shock effects, and the macroscopic failure phenomena of the tunnels under top and side explosion loads, and pointed out the difference of antiexplosion performance of spray-anchor supporting tunnels in sandy gravel stratum under the two different explosion loads.

Currently, there are two major approaches for researching the anti-explosion capacity of anchorage tunnels, namely, experimental research and numerical simulation. However, numerical simulation is an indirect method in the failure analysis of anchored tunnel; furthermore, appropriate constitutive models and accurate parameters of materials are critically important to the reliability of numerical simulation results. Many field tests are not feasible because of safety and economic reasons. Besides, the preparation and measurement in full-scale field experiments are extremely complex and expensive. In contrast, small-scale model tests, which can be used to identify the critical influence factors, optimize engineering design and establish dynamic response models of engineering, have many obvious advantages, such as short cycle, low cost, relatively simple measurement layout, and intuitive experimental phenomena [18-19]. Many small-scale model tests have been conducted by some scholars. Wang et al. [20] carried out the model tests to study the anti-explosion capacity of two tunnels reinforced by wave absorbing bolts and dense bolts set up at inner anchoring sections, respectively. Test results showed that the fractures in the vault were obviously decreased and the damage degree of tunnels was obviously reduced.
The above researches mainly involve the propagation of blast wave in the surrounding rock of the tunnel, the mechanical response of the bolt under the action of blast wave, the destruction of the tunnel under specific explosion load and supporting measures, etc. However, due to the complexity of the wave problem, the limitation of the field test conditions and the unsystematic model test, there are so few studies on the failure modes of the anchorage tunnel at present, that can not well guide the anti-explosion reinforcement design of the tunnel, and it is difficult to put forward the reasonable and effective measures for the reinforcement.

In view of the shortcomings of existing research, based on Froude similarity theory, a series of anti-explosion model tests on different anchorage tunnels subjected to blast loads, including plane charge and mass charge, were carried out in this study. The failure modes of several kinds of anchorage tunnels under explosive loading were obtained and the failure law and failure mechanism were analyzed. This study lays a foundation for improving the anti-explosion measures and enhancing the anti-explosion ability of underground tunnels.

The remainder of this study is organized as follows. Section 3 described the model test similarity theory, the test device, the test models and the explosion load simulation methods. Section 4 analyzed the anti-explosion test results of different schemes reinforced tunnels. Conclusions are summarized in Section 5.

\section{Methodology}

\subsection{Similarity theory}

At present, two sorts of similarity theories have been widely used in model tests, namely replication similarity theory and Froude similarity theory. The similar scaling factors of the two similarity theories are shown in table 1.

In replica scaling, the same material is used for model and prototype, and the scaling laws indicate that the acceleration scale factor should be the reciprocal of the length scale factor. This requirement can be satisfied by using a centrifuge.

In Froude scaling, the set of base variables consists of length, mass density and acceleration. The acceleration is chosen as a base variable and is assigned a scale factor of unity. For systems in which gravity effects are important this method can be employed to design model tests without requiring the use of a centrifuge. When Froude scaling is used, the properties of the model or simulant material need to satisfy the following scaling laws: stress $\sigma=\rho l$, strain $\varepsilon=1$, Poisson's ratio $\nu=1$, and friction angle $\varphi=1$.

As a result, the prototype materials cannot be used to construct small-scale models; instead simulant materials should be substituted for prototype materials. Because the product of the length and mass density scale factors is usually less than 1 , the simulant materials need to be weaker than the prototype materials.

In 1988 T.F. Zahrah conducted a model test study on the dynamic responses of the shallow buried arch subjected to conventional weapon by using Froude scaling, in which the length scale factors are $1 / 5,1 / 18,1 / 50$, respectively. Test results confirmed that small-scale explosion model tests using Froude scaling are reliable. Zhang et al. [21] also carried out explosion model tests based on Froude similarity theory, and compared them with the prototype tests. These regularities for the displacement and acceleration of tunnel 
Xu Jingmao, Gu Jincai, Chen Anmin, Zhang Xiangyang and Wang Tao/

Journal of Engineering Science and Technology Review 11 (4) (2018) 118 - 125

wall, the stain of bolts, and the failure modes of tunnels between model and prototype were similar, fully proving that it is feasible to carry out small-scale explosion model test by using Froude scaling, and the test result is reliable. So in this study, small-scale model tests were conducted by using simulant materials based on Froude similarity theory.

Table 1. Similar scaling factors

\begin{tabular}{c|c|c|c}
\hline Variable & Scale Factor & Froude Scale Factor & Replica Scale Factor \\
\hline Length & $l$ & $l$ & $l$ \\
Mass density & $\rho$ & $\rho$ & $\rho=1$ \\
Acceleration & $a$ & $a=1$ & $a=1 / l$ \\
Time & $t=(l / a)^{1 / 2}$ & $t=l^{1 / 2}$ & $t=l$ \\
Stress & $\sigma=\rho a l$ & $\sigma=\rho l$ & $\sigma=1$ \\
Velocity & $v=(a l)^{1 / 2}$ & $v=l^{1 / 2}$ & $v=1$ \\
Force & $f=\rho a l^{3}$ & $f=\rho l^{3}$ & $f=l^{2}$ \\
Unit weight & $\gamma=\rho a$ & $\gamma=\rho$ & $\gamma=1 / l$ \\
Impulse & $i=\rho\left(a l^{3}\right)^{1 / 2}$ & $i=\rho l^{3 / 2}$ & $i=l$ \\
Energy & $w=\rho a l^{4}$ & $w=\rho l^{4}$ & $w=l^{3}$ \\
\hline
\end{tabular}

Note: Each italic letter in the table does not represent a variable, but the scale factor of the corresponding variable. The scale factor for a variable is defined as the ratio of its model to prototype values. For example, the scale factor for length, $l$, is given by $l_{m} / l_{p}$, where the subscripts $m$ and $p$ refer to model and prototype, respectively. The scale factor for dimensionless quantities, such as strain, Poison's ratio, and friction angle, is unity.

\subsection{Test device}

In this study, the model tests were completed by using the model test apparatus for anti-explosion structures in geotechnical engineering [22], which is shown in Fig. 1. It is composed of 4 box walls and 1 Foundation pit. The size of the foundation pit is $2.4 \mathrm{~m} \times 1.5 \mathrm{~m} \times 0.5 \mathrm{~m}$ (length, width, and depth) and 4 box walls are situated around it. The 4 box walls can move along the track before and after by the horizontal tensioning jacks mounted on their backs, and by screw connections to form a test box with an upper opening (see Fig. 1(a)), in which model can be made and explosion can be conducted. After explosion test, the box walls can be pulled apart (see Fig. 1(b)), so as to observe and process the test model. In order to meet the stiffness and strength requirements of the test box, the box wall is made of $10 \mathrm{~mm}$ thick steel plate, and strengthened by channel No. 30 and steel fiber concrete from inside. The cross section of the box wall is trapezoidal, with a top width of $0.4 \mathrm{~m}$, a bottom width of $0.8 \mathrm{~m}$ and a height of $1.8 \mathrm{~m}$.

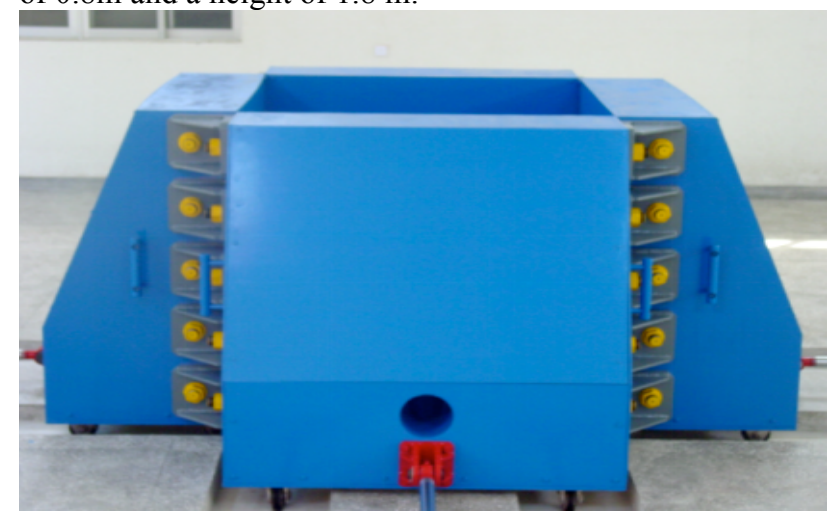

(a) Gathered together

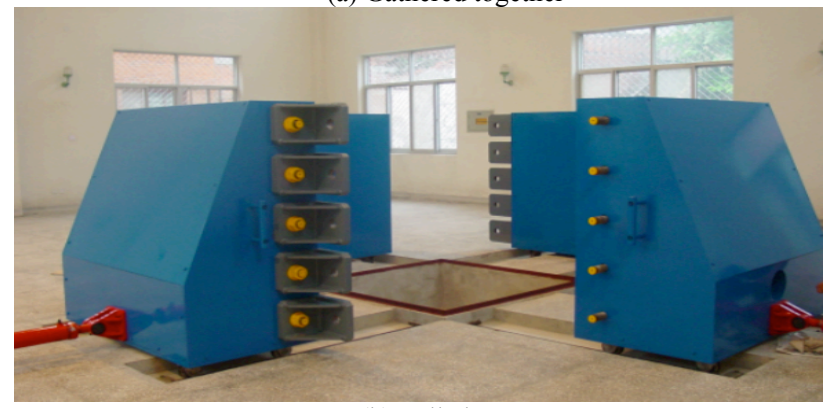

(b) Pulled apart

Fig. 1. Model test apparatus for anti-explosion structures in geotechnical engineering

\subsection{Test model and similarity scaling}

The structural dimensions of the test model are shown in Fig. 2. The simulate tunnel is straight wall and vault in shape, and its axis orientation is along $1500 \mathrm{~mm}$ length.

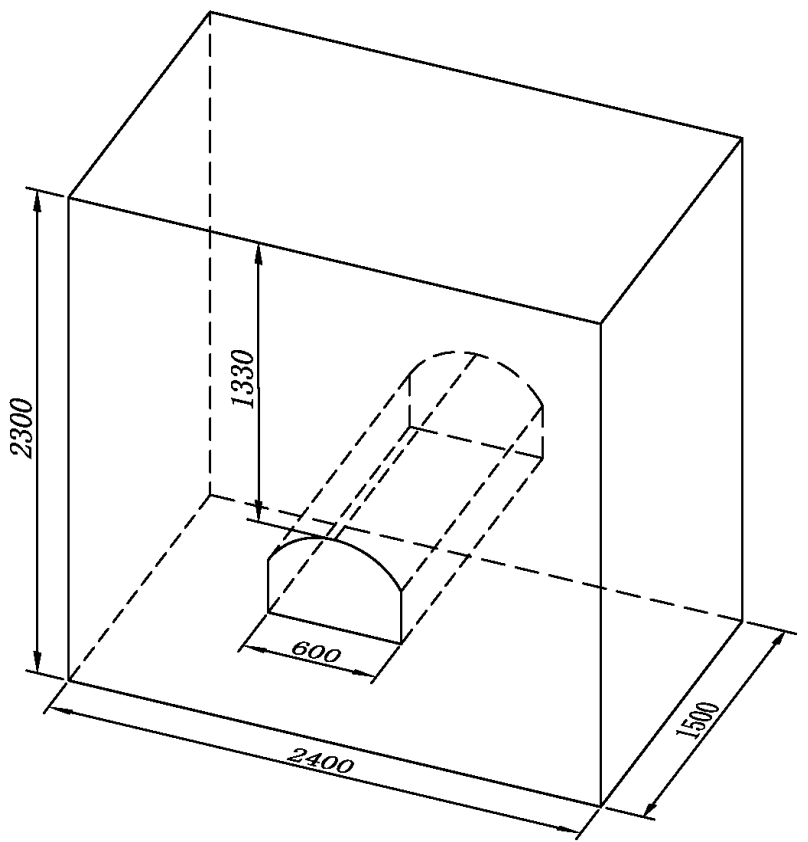

Fig. 2. Structural dimensions of test model (mm)

According to Froude similarity theory, the scale factors of the model test are determined. The rock quality of the prototype is grade 2 to 3 , and the span, wall height, and arc height of tunnel are $6 \mathrm{~m}, 2.5 \mathrm{~m}$, and $1.7 \mathrm{~m}$, respectively. Considering a variety of factors generally, the length, mass density, and stress scale factors are assigned $0.1,0.75$, and 0.075 , respectively. The scale factors for other variables can be obtained by using the fundamental scaling law. It's noted that the loading similitude requirements can be satisfied in part, the similitude requirement for either peak pressure or for impulse, but not both can be satisfied. In general, if the maximum response of the system occurs early, peak pressure should be properly scaled; if the maximum response of the system occurs after overpressure has decayed substantially, the impulse should be properly scaled. In the model test, the peak relative displacement between vault and floor is selected as the criterion of the maximum response of the 
Xu Jingmao, Gu Jincai, Chen Anmin, Zhang Xiangyang and Wang Tao/

Journal of Engineering Science and Technology Review 11 (4) (2018) 118 - 125

system. The measured data show that the peak relative displacement between vault and floor occurs before pressure has decayed substantially. Therefore, the explosive charge in the model is determined according to that the similitude requirement for peak pressure is satisfied.
In the test, the low-grade cement mortar is chosen as the simulant material of rock, and its physical and mechanical parameters are shown in table 2. The simulant material of bolt is aluminium wire and the simulant material of grouting is gypsum slurry.

Table 2. Physical and mechanical parameters of model material

\begin{tabular}{c|c|c|c|c|c|c|c}
\hline Parameter name & $\rho\left(\mathrm{kg} / \mathrm{m}^{3}\right)$ & $R_{c}(\mathrm{MPa})$ & $R_{t}(\mathrm{kPa})$ & $E(\mathrm{GPa})$ & $c(\mathrm{kPa})$ & $\varphi\left({ }^{\circ}\right)$ & $w(\%)$ \\
\hline Prototype material & $2250-2650$ & $15.000-30.000$ & $750-3000$ & $6.000-30.000$ & $700-2100$ & $39-50$ & - \\
Required material & $1688-1988$ & $1.125-2.250$ & $56-225$ & $0.450-2.250$ & $53-159$ \\
Model material & 1797 & 1.827 & 176 & 1.832 & $39-50$ & 115 \\
\hline
\end{tabular}

\subsection{Explosion mode}

In the model test, there are two kinds of explosion modes, including plane charge and mass charge. The plane charge adopts the reticulate detonating cords arranged parallel with the tunnel axis, and the mass charge adopts blocky TNT. In these two ways, the explosive charges are buried in the medium of a certain depth above the tunnel vault. The plane charge explosion requires that roughly uniform compressive stress waves occur in the same horizontal plane above the tunnel vault. The stress measure points $P_{1}-P_{5}$ given in Fig. 3(a) are arranged in the same horizontal plane above the tunnel vault, and their distribution range is 2.4 times of the tunnel span. The minimum peak pressure is $0.33 \mathrm{MPa}$ and the maximum peak pressure is $0.38 \mathrm{MPa}$ (Fig. 3(b)).

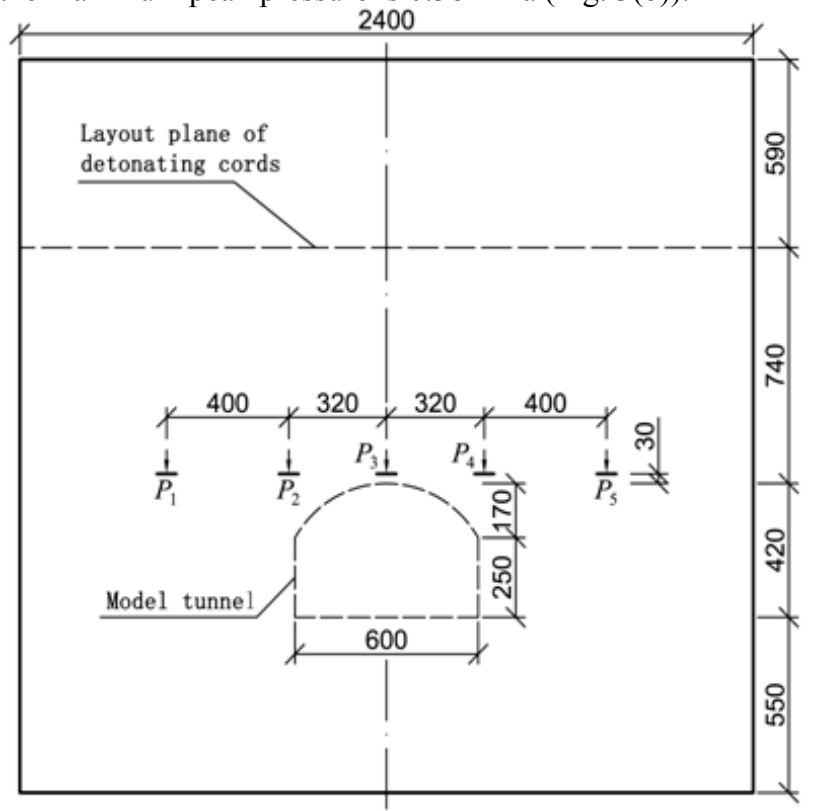

(a) Pressure measurement points arrangement (mm)

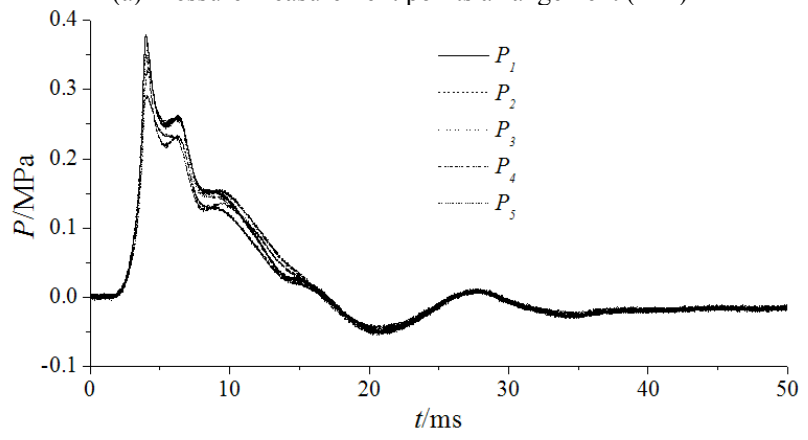

(b) Measured pressure waveforms

Fig. 3. The pressure waves in the same horizontal plane above the tunnel vault in the plane charge explosion

\section{Result analysis and discussion}

\subsection{Comparison of failure modes of anchorage tunnels} under plane charge explosion

Under the plane charge explosion, the test conditions of anchorage tunnels are as follows. The model tunnels are all in the shape of straight wall vault with a span of $60 \mathrm{~cm}$, a height of $42 \mathrm{~cm}$, a wall height of $25 \mathrm{~cm}$ and an arch height of $17 \mathrm{~cm}$. The simulant material of rock is cement mortar, and the occurrence is layered block. The explosive charge is the same, namely $W=391 \mathrm{~g}$ TNT; the buried depth of the charge $H e=59 \mathrm{~cm}$; the distance between explosion center and vault $R=122 \mathrm{~cm}$. Under the above conditions, the failure modes of each model tunnel are as follows:

\subsubsection{Failure modes of unreinforced model tunnel}

The damage pattern of the unreinforced model tunnel is shown in Fig. 4. It can be seen from the figure that large blocks of material fall off the surface of the vault in the unreinforced model tunnel, but there is no obvious dislocation crack in the spandrels on both sides of the tunnel.

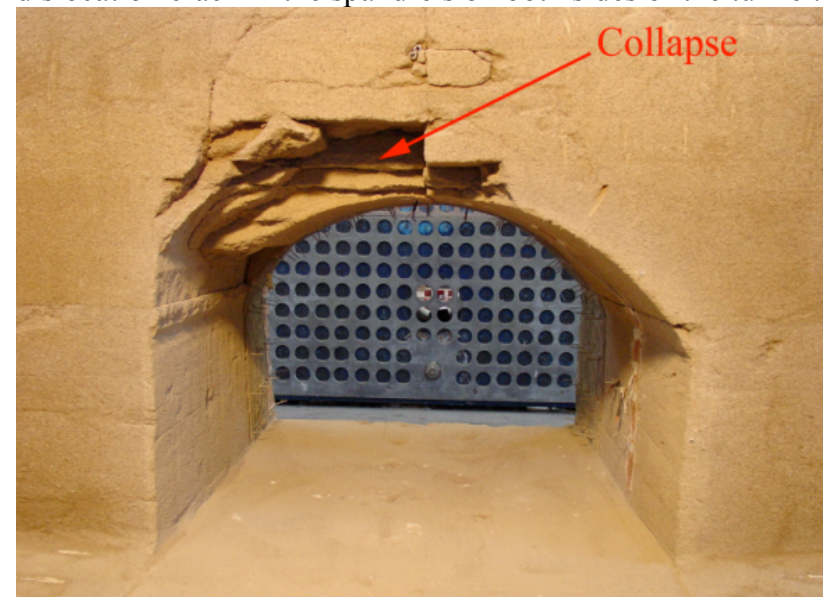

Fig. 4. Failure modes of unreinforced model tunnel

4.1.2 Failure modes of the model tunnel reinforced by partgrouted bolts

The medium and explosion conditions of this model are the same as those of the unreinforced tunnel model. The difference is that the model tunnel in this model is reinforced by part-grouted bolts. The simulant bolt is an aluminum wire with a diameter of $2.1 \mathrm{~mm}$ and a length of $20 \mathrm{~cm}$. The free length $L_{f}$, anchorage length $L_{a}$, and spacing $a$ of the simulant bolt are $13 \mathrm{~cm}, 7 \mathrm{~cm}, 6.7 \mathrm{~cm}$, respectively. The $\mathrm{M} 2$ nut is fixed on the simulant anchor plate, which is made of aluminum plat with $2 \mathrm{~mm} \times 12 \mathrm{~mm} \times 12 \mathrm{~mm}$. In addition, in order to strengthen the connection between bolts, the reinforcement plates are arranged along two directions (axial and transverse) of outer fixed ends of the adjacent bolts, 
Xu Jingmao, Gu Jincai, Chen Anmin, Zhang Xiangyang and Wang Tao/

Journal of Engineering Science and Technology Review 11 (4) (2018) 118 - 125

whose material is the beryllium bronze with $0.3 \mathrm{~mm}$ thick and $10 \mathrm{~mm}$ wide.

Under the above conditions, the failure characteristics of the model tunnel (Fig. 5) are that the large rock mass in the vault is loose without dropping. The loosening range is similar to that of the unreinforced model tunnel, indicating that the bolt-anchor plate-reinforcement plate system plays an obvious role in preventing the rock block from falling off. There is also no obvious dislocation crack in the spandrels.

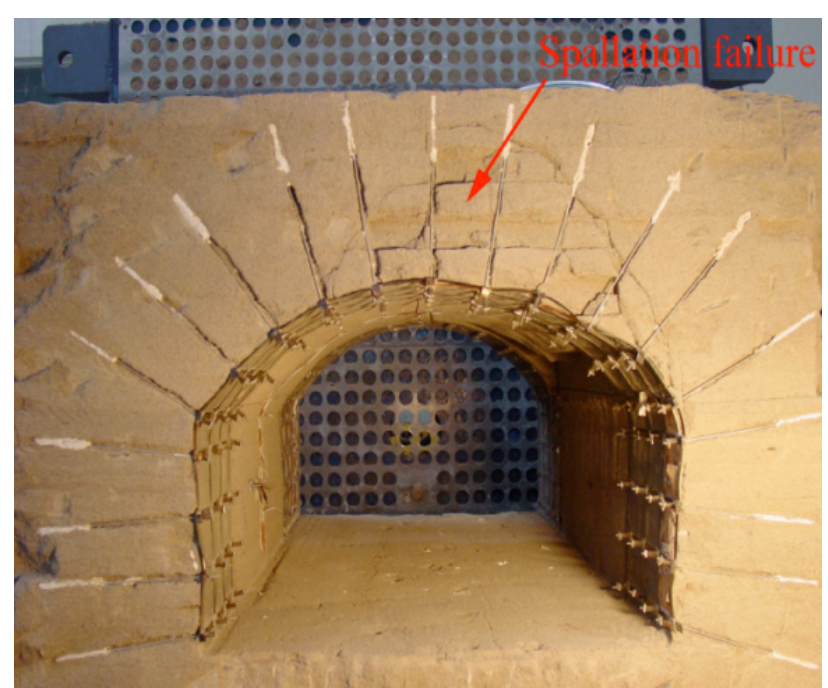

Fig. 5. Failure modes of the model tunnel reinforced by part-grouted anchor bolts

\subsubsection{Failure modes of the model tunnel reinforced by} full-bonded bolts

In addition to the full bonded bolt in this model, namely $L_{a}=20 \mathrm{~cm}$, the other conditions are the same as those of the model tunnel reinforced by part-grouted bolts. Under the above conditions, the fracture characteristics of this model tunnel are shown in Fig. 6. It can be obtained from Fig. 6 that, under the same explosion load, the damage degree of the model tunnel reinforced by full-bonded bolts is much slighter than that by part-grouted bolts. It is only above the vault that a few fine cracks occur, and the spalling failure does not appear. The apparent difference in the damage degree clearly indicates that the reinforcement of the fullbonded bolts is much more effective than the part-grouted bolts.

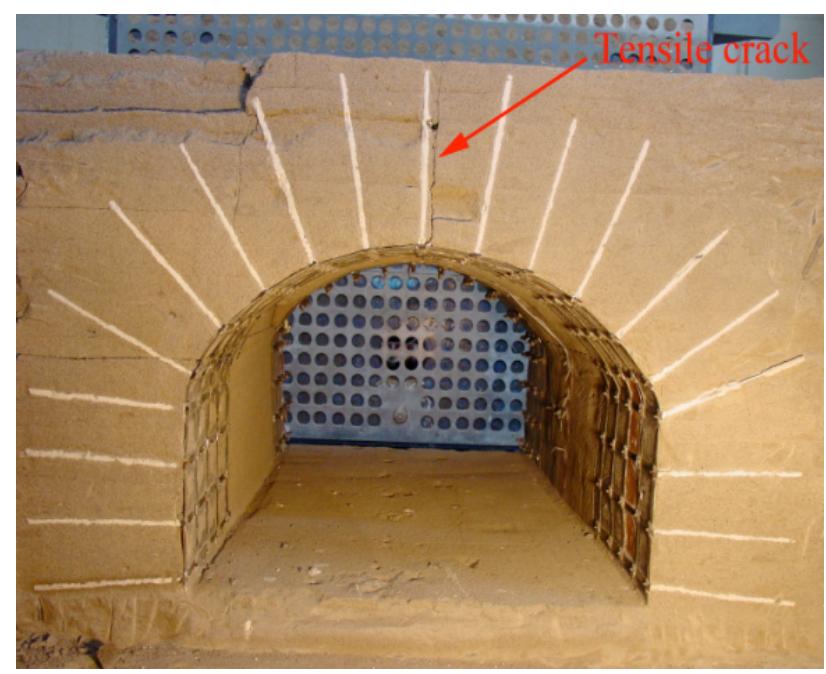

Fig. 6. Failure modes of the model tunnel reinforced by full-bonded bolts
4.2 Comparison of failure modes of anchorage tunnels under mass charge explosion

Under the mass charge explosion, the simulant material of rock is the uniform cement mortar instead of the layered one. The shape and size of the model tunnels are the same as that of plane charge. The bolts in this group models are all full bonded with different bolt support parameters, which are shown in the specific layout of each model.

In order to facilitate the comparison of failure modes between different anchorage tunnels, in addition to different bolt support parameters, there are the certain same basic parameters, such as the shape and size of the model tunnel, the explosive charge ( $W=100 \mathrm{~g}$ TNT), the buried depth of the charge $(H e=85 \mathrm{~cm})$, the distance between explosion center and vault $(R=48 \mathrm{~cm})$.

Under the above conditions, the failure modes of each model tunnel are as follows:

\subsubsection{Failure modes of the model tunnel reinforced by common bolts}

The bolt support parameters in this model are as follows: the length, $L=18 \mathrm{~cm}$, the spacing, $d=6 \mathrm{~cm}$, the diameter, $\phi=1.84$ $\mathrm{mm}$, and the simulant material of bolts is also aluminium wire. To make it easy to distinguish, they are called as common bolts.

The fracture modes of the model tunnel are shown in Fig. 7. As can be seen from Fig. 7, the damage characteristic is that, one or more oblique shear cracks occurs in the medium above the spandrels on both sides of the tunnel, and the cracks run downward from the explosion center into a bell shaped, some cracks directly come through the bolts, and other cracks occur in the medium that outside the reinforced region. The above phenomena show that common bolt support cannot prevent shear cracks from extending into the reinforced region, and cracks can extend cross bolts.

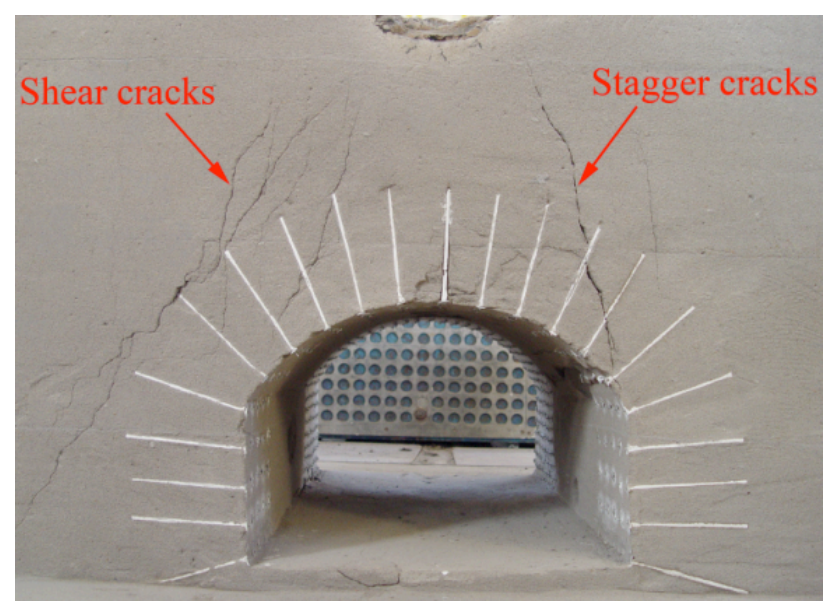

Fig. 7. Failure modes of the model tunnel reinforced by common bolts

4.2.2 Failure modes of the model tunnel reinforced by long-dense bolts

Except that the bolt spacing in this model is reduced from 6 $\mathrm{cm}$ to $3 \mathrm{~cm}$, the other bolt parameters are the same as those of the model tunnel reinforced by common bolts. They are called as long-dense bolts. The fracture characteristics of this model tunnel are shown in Fig. 8. Compared with Fig. 7, although the two model tunnels all have a number of oblique cracks in the medium above the spandrels, which can be seen from Fig. 8, the cracks in this model all occur outside the length range of bolts, and there is no crack in the reinforced region. This shows that the decrease of the bolt spacing can prevent the penetration of cracks. It is an important inspiration for seeking technical measures to 
Xu Jingmao, Gu Jincai, Chen Anmin, Zhang Xiangyang and Wang Tao/

Journal of Engineering Science and Technology Review 11 (4) (2018) 118 - 125

improve the anti-explosion ability of anchorage tunnels.

\subsubsection{Failure modes of the model tunnel reinforced by short-dense bolts}

Except that the bolt length in this model is reduced from 18 $\mathrm{cm}$ to $6 \mathrm{~cm}$, the other bolt parameters are the same as those of the model tunnel reinforced by long-dense bolts. They are called as short-dense bolts. The fracture characteristics of this model tunnel are shown in Fig. 9. It can be obtained from Fig. 9, one or more oblique cracks occurs in the medium above the spandrels on both sides of the tunnel, and cracks also occur outside the length range of bolts without extending into the reinforced region. However, the cracks are closer to the wall than those in the long-dense support tunnel, so there is a larger risk.

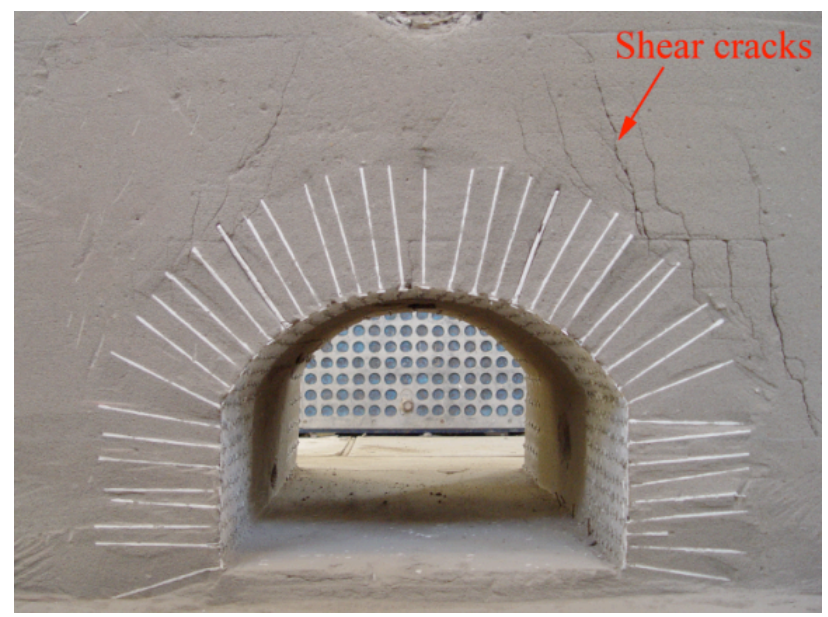

Fig. 8. Failure modes of the model tunnel reinforced by long-dense bolts

4.2.4 Failure modes of the model tunnel reinforced by long-short alternation bolts

The bolt parameters are basically the same as those in Fig. 9, but only one long bolt is placed among three short bolts, that is to say, the original bolt length $L=6 \mathrm{~cm}$ is changed to $L=18$ $\mathrm{cm}$. They are called as long-short alternation bolts. The fracture characteristics of this model tunnel are shown in Fig. 10. It can be obtained from Fig. 10, compared with shortdense bolts in Fig. 9, a few long bolts are added to prevent the emergence and development of shear cracks, but there is no obvious effect, and cracks go cross the long bolts without any change.

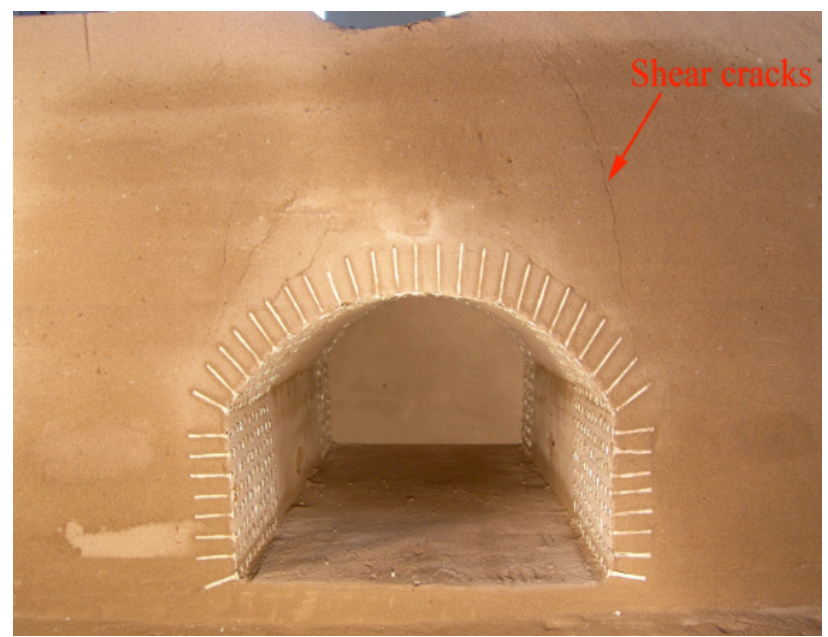

Fig. 9. Failure modes of the model tunnel reinforced by short-dense bolts
4.2.5 Failure modes of the tunnel reinforced by longdense and local lengthening bolts

In view of shear cracks that occur outside the reinforced region of spandrels in Fig. 8, this model test is conducted to investigate the change of shear cracks under the condition that the bolt length is lengthened. In this model, the bolt parameters are basically the same as those in Fig. 8, but the length of 6 rows of bolts in the spandrel is lengthened to 30 $\mathrm{cm}$ (formerly $18 \mathrm{~cm}$ ). The fracture characteristics of this model tunnel are shown in Fig. 11.

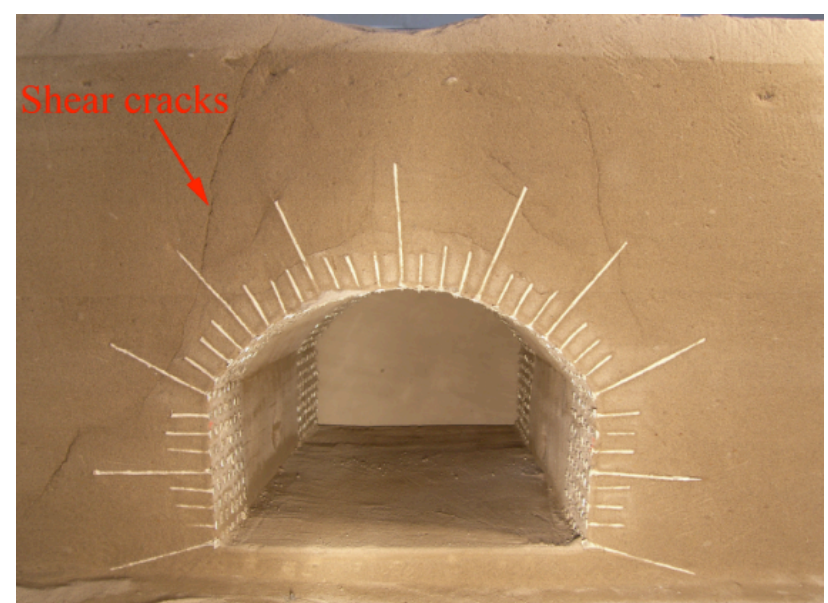

Fig. 10. Failure modes of the model tunnel reinforced by long-short alternation bolts

As it can be seen from Fig. 11, the lengthened bolts in spandrels effectively prevent the extension of shear cracks, that is to say, the cracks are interdicted by local lengthened bolts. Secondly, in the view of the number of cracks and the degree of cracking, it is also less and slighter than that of the model tunnel reinforced by common bolts (Fig. 8), showing that this reinforcement scheme is beneficial to improve the resistance of the tunnel.

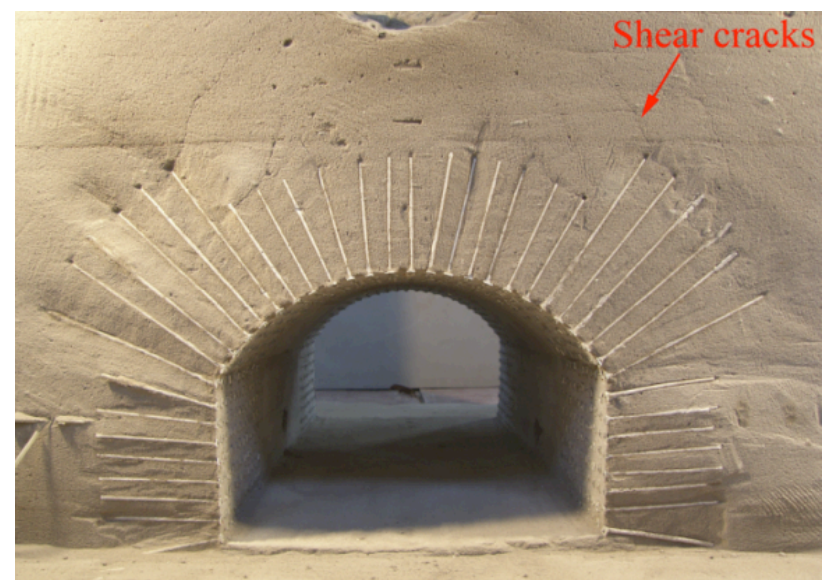

Fig. 11. Failure modes of the model tunnel reinforced by long-dense bolts and local lengthening bolts in spandrels

\subsubsection{Failure modes of the tunnel reinforced by short-} dense and local lengthening bolts

It is the same reason to carry out this model test as that shown in Fig. 11. In this model, the bolt parameters are basically the same as those in Fig. 9, but the length of 6 rows of short bolts in the spandrel is lengthened to $18 \mathrm{~cm}$ (formerly $6 \mathrm{~cm}$ ). The fracture characteristics of this model tunnel are shown in Fig. 12.

It can be seen from the Fig. 12, the shear cracks in the surrounding rock are also interdicted by local lengthened short-bolts in spandrels, but there are still cracks outside the 
Xu Jingmao, Gu Jincai, Chen Anmin, Zhang Xiangyang and Wang Tao/

Journal of Engineering Science and Technology Review 11 (4) (2018) 118 - 125

length range of local lengthened short-bolts. In addition, the cracking degree is obviously slighter, which shows that this reinforcement scheme can also improve the resistance of the tunnel.

In addition, in this group model tests, it is found that spalling failure does not occur in tunnel vault, and there are only one or more shear cracks in the medium above the spandrels. This is obviously different from the damage characteristics of the model tunnels under plane charge explosion, which is caused by the nonuniform stress distribution in the same horizontal plane above tunnel vault by mass charge. The maximum stress distribution is situated under the explosion center, and the stress decrease with the increase of distance from the explosion center. Meanwhile, the vault is located below the explosion center, which is the free surface without supporting force, resulting in larger downward displacement of the vault. The two sides of the tunnel vault are supported by the side walls, so the downward displacement is smaller. This inevitably causes uncoordinated displacement between the vault and the side walls, resulting in one or more shear cracks in the spandrels. However, under the plane charge explosion condition, the more uniform plane pressure is generated above the vault; the pressure difference between the vault and the side walls is less than that of the mass charge explosion condition. Therefore, no shear dislocation cracks appear in the spandrels on neither of the tunnel side, but spalling or tensile cracks occur in the vault.

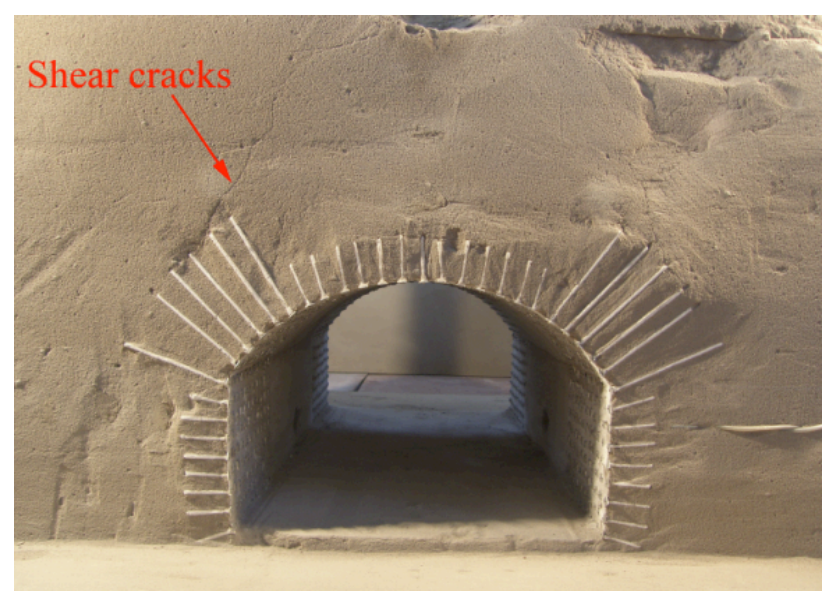

Fig. 12. Failure modes of the model tunnel reinforced by short-dense bolts and local lengthening bolts in spandrels

\section{Conclusions}

To obtain the failure modes of underground anti-explosion tunnels and to guide the design of anti-explosion reinforcement scientifically, based on Froude similarity theory, the anti-explosion model tests of unreinforced and reinforced tunnels under plane charge and mass charge were carried out in this study. The failure rules of several kinds of anchorage tunnels were analyzed, and the main conclusions are as follows:

(1) There are obvious differences in the failure modes of the anchorage tunnels under the plane charge explosion and the mass charge explosion. Under the plane charge explosion, almost no shear cracks appear in the spandrels and only spalling occurs in the vault. The mass charge explosion will cause oblique shear cracks in the medium above the spandrels on both sides of the anchorage tunnel, but no spalling failure in the vault. This shows that the failure modes of the anchorage tunnels are different in different explosion modes.

(2) The full-grouted bolt has better anti-explosion effect than the part-grouted bolt, so the anti-explosion engineering should be reinforced by full-grouted bolts instead of partgrouted bolts.

(3) When the bolt length and spacing are large, it has little effect on preventing the occurrence and extension of the shear cracks. Only the bolt spacing is small to a certain extent, it can prevent the shear cracks from penetrating into the reinforced region.

(4) Local lengthened dense-bolts in the spandrels can prevent the extension of the shear cracks, compel cracks to detour the reinforced region, or only appear outside their length range. This reinforcement scheme can sharply reduce the cracking degree of surrounding rock fracture, so it can improve the anti-explosion capability of the anchorage tunnel.

The anti-explosion failure modes of anchorage tunnels obtained by this study are systematic and comprehensive, which lay a foundation for taking targeted anti-explosion reinforcement measures and greatly improving antiexplosion ability of underground tunnels. However, under the condition of economic permissibility, field test research should also be carried out, so as to provide a practical basis for a more comprehensive understanding of the failure law of anti-explosion tunnels.

This is an Open Access article distributed under the terms of the Creative Commons Attribution License

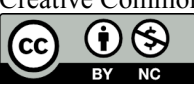

\section{References}

1. Cheng, L. K., "Present status and development of ground anchorages". Civil Engineering Journal, 34(3), 2001, pp. 7-12.

2. Gu, J. C., Chen, A. M., "Research on prospect of rock reinforcement technique". Tunnel Construction, 24(1), 2004, pp. 1-25.

3. Jin, F. N., Liu, L., Zhang, L. P., Li, B., Fan, H. L., "Development and penetration of deep drilling ground weapons". Journal of PLA University of Science and Technology (Natural Science edition), 3(2), 2002, pp. 34-40.

4. Wang, T., Yu, W. L., Wang, S. L., Quan, W., "Status and development tendency of foreign earth-penetrating weapons". Missiles and Space Vehicles, 278(5), 2005, pp. 51-56.
5. Wu, J., Deng, K., Liu, S. K., "American precisely-guided weapon and its countermeasures analysis". Winged Missiles Journal, 6, 2007, pp. 12-16.

6. Yang, H., Wang, C., "Study on propagation law of Elastic wave in anchorage system". Journal of test and measurement technology, 17(2), 2003, pp. 145-149.

7. Tannant, D. D., Brummer, R. K., Yi, X., "Rockbolt behavior under dynamic loading field tests and modeling". International Journal of Rock Mechanics and Mining Sciences and Geomechanics Abstracts, 32(6), 1995, pp. 537-550.

8. Yang, S. H., Liang, B., Gu, J. C., Shen, J., Chen, A. M., "Research on characteristics of prestress change of anchorage cable in anti- 
Xu Jingmao, Gu Jincai, Chen Anmin, Zhang Xiangyang and Wang Tao/

\section{Journal of Engineering Science and Technology Review 11 (4) (2018) 118 - 125}

explosion model test of anchored cavern". Chinese Journal of Rock Mechanics and Engineering, 25(S2), 2006, pp. 3750-3756.

9. Ortlepp, W. D., Stacey, T. R., "Performance of tunnel support under large deformation, static and dynamic loading". Tunnelling and Underground Space Technology, 13(1), 1998, pp. 15-21.

10. Yi, C. P., Lu, W. B., "Research on influence of blasting vibration on grouted rockbolts". Rock and Soil Mechanics, 27(8), 2006, pp. 1312-1316.

11. Zhang, C. S., Zou, D. H., Madenga, V., "Numerical simulation of wave propagation in grouted rock bolts and the effects of mesh density and wave frequency". International Journal of Rock Mechanics and Mining Sciences, 43(4), 2006, pp. 634-639.

12. Xue, Y. D., Zhang, S. P., Kang, T. H., "Numerical analysis of dynamic response of rock bolts in mining roadways". Chinese Journal of Rock Mechanics and Engineering, 22(11), 2003, pp. 1903-1906.

13. Rong, Y., Xu, X. B., Zhao, M. J., Huang, H. Y., "Numerical simulating propagation of stress waves in the country rock with bolting". Chinese Journal of Underground Space and Engineering, 2(1), 2006, pp. 115-119.

14. Zeng, X., M., Du, Y., H., Li, S. M., "Testing study of comparison between prototype and model on resisting dynamical load with soil nail supporting". Chinese Journal of Rock Mechanics and Engineering, 22(11), 2003, pp. 1892-1897.

15. Xiao, F., Zeng, X. M., "Study on blast-resistant performance of loess tunnel with shotcrete-bolt support- Distributional pattern of ambient pressure". Protective Engineering, 13(4), 1991, pp. 37-45.

16. Cao, C. L., Zeng, X. M. "Study on blast-resistant performance of loess tunnel with shotcrete-bolt support- Mechanical and deformation characteristics of support; Critical bearing capacity". Protective Engineering, 14(1), 1992, pp. 46-55.

17. Ren, H. Q., "Study on anti-explosion performance of spray-anchor supporting tunnels in sandy gravel stratum under top and side explosion loads". Protective Engineering, 8(1), 1986, pp. 12-18.

18. Wang, S. R., Li, D. J., Li, C. L., Zhang, C. G., Zhang, Y. B. "Thermal radiation characteristics of stress evolution of a circular tunnel excavation under different confining pressures". Tunnelling and Underground Space Technology, 78, 2018, pp. 76-83.

19. Wang, S. R., Li, C. L., Li, D. J., Zhang, Y. B., Hagan, P., “Skewed pressure characteristics induced by step-by-step excavation of double-arch tunnel based on infrared thermography". Tehnicki Vjesnik-Technical Gazette, 23(3), 2016, pp. 827-833.

20. Wang, G. Y., Gu, J. C., Chen, A. M., Xu, J. M., Zhang, X. Y., "Model test research on anti-explosion capacity of underground openings with end wave-decay by holes and reinforced by dense rock bolts". Chinese Journal of Rock Mechanics and Engineering, 29(1), 2010, pp. 51-58.

21. Zhang, X. Y., Gu, J. C., Shen, J., Xu, J. M., Chen, A. M., Ming, Z. Q., "The comparison of the results of model test and field test on anchored caverns under explosion loading". Protective Engineering, 34(1), 2012, pp. 6-12.

22. Shen, J., Gu, J. C., Chen, A. M., Xu, J. M., Ming, Z. Q., Zhang, X. Y., "Development and applications of the model test apparatus on anti-explosion structures in geotechnical engineering". Chinese Journal of Underground Space and Engineering, 3(6), 2007, pp. 1077-1080. 